\title{
Survey on Iris Based Recognition Systems
}

\section{Author: Shashikala HK ${ }^{1}$; Shreya Nupur Shakya²; Pooja Panjiyar³; Abhinav Singh Upreti ${ }^{4}$ Shaik Dadapeer5;}

\author{
Affiliation: Jain University ${ }^{1}$; Jain University ${ }^{2}$; Jain University ${ }^{3}$; Jain University4; Jain University ${ }^{5}$ \\ E-mail: shashi.hk85@gmail.com ${ }^{1}$; 17btrcs141@jainuniversity.ac.in² \\ 17btrcs076@jainuniversity.ac.in ${ }^{3} ; 17 b t r c s 135 @ j a i n u n i v e r s i t y . a c . i n^{4}$; \\ 17btrcs120@jainuniversity.ac.in ${ }^{5}$
}

DOI <10.26821/IJSHRE.8.12.2020.81216>

\section{ABSTRACT}

In today's tech-savvy educational institutions, attendance is considered as an important factor for both students and institutional authorities. However, professors still have to go through manual procedures to record attendance which is rather slow and inefficient. Iris recognition verification is a reliable personal identification method used in biometric identification. Among all biometric methods, the automated personal identity authentication systems based on iris recognition is known to be the most reliable one. With the increase in development of iris recognition systems, there have been a number of proposals for various applications, including the attendance management system. Additionally, issues regarding proxy attendance, inaccuracy and students' unawareness of their attendance status arise undeniably. To the best of our knowledge, no Iris based recognition system has been implemented in our university for the purpose of attendance monitoring and information retrieval. The proposed project aims at developing a system which will facilitate professors in attendance monitoring of a classroom. This project also aspires to build flexibility for students to view their own information, attendance status, faculty information and timetable. The professors will also be able to view the attendance status of every student, information of all students and their own daily schedule. When fully implemented, we believe the system will assist in changing the traditional attendance management system into an accurate and efficient system. The system will also provide flexibility to the users to retrieve certain permitted information. Moreover, the system will utilize and implement concepts of deep learning to make a better system compared to the existing ones.
\end{abstract}

Keywords: iris recognition, attendance monitoring, artificial intelligence, computer vision, biometrics, iris detection

\section{INTRODUCTION}

As the digital era is uplifting at a very high rate, biometrics technologies have begun to have a greater effect on the everyday lives of people more and more. In biometric systems there exist different types of biometric such as thumb recognition, palm recognition, face recognition and iris recognition. Iris recognition systems are one of the most mature and popular biometrics systems used in the field of automatic personal identification. It is a real-time recognition of a person's identity that makes use of mathematical analysis of the random patterns that can be seen inside the iris of an eye from some distance. The iris of the human eye is a protected internal organ with a complex texture and it is very stable throughout our lifetime. In other words, it can serve as a living passport that one need not remember but is always present. Iris Scans are faster and more precise than fingerprint scans as it is comparatively easier for an individual to screen their eyes than it is to adjust their fingers. Iris recognition is an important application of biometric artificial intelligence owing to its use in many fields. Identification of individuals in an institution for the purpose of attendance is one such application of iris recognition. In the analysis of performance of any organization, maintenance and monitoring of attendance records play a significant role. The purpose of developing an automated system for attendance management is to computerize the traditional way of taking attendance in educational institutions across India. This system aims at performing the daily activities of traditional attendance marking and

Shashikala HK; Shreya Nupur Shakya; Pooja Panjiyar; Abhinav Singh Upreti; Shaik Dadapeer, Volume 8

Issue 12, pp 59-62, December 2020 
Volume 8 Issue 12 December 2020

attendance analysis with reduced human intervention. The prevalent techniques and methodologies for detecting and recognizing other biometrics fail to overcome issues such as physical touch, increased time, high cost and inefficiency. The proposed system also aims at overcoming the existing pitfalls of the current systems along by supporting with features such as detection of iris and analysis of students' attendance. The system uses algorithms that localize the iris, perform pattern matching and perform feature extraction for detection and recognition of the irises.

\section{Related Works}

This section will focus on the works related to various systems using iris recognition.

\subsection{Implementation of the Hough Transform for Iris Detection and Segmentation}

\subsubsection{Proposed Technique \&Features}

The technique suggested consists of six main phases: image acquisition, pre-processing, localization of the iris, edge localization, extraction of the iris, and postprocessing. This project implements a Hough transform for iris detection due to the circular structure of the iris.

\subsubsection{Advantages}

It uses Hough transform to complete the external circumference of the regularly used images of the database.

\subsubsection{Limitations}

The appearance and the distance of circular objects within an iris image make it difficult to locate the iris.

Factors such as distance and lighting can affect the accuracy of the system.

\subsection{Texture Analysis Based Iris Recognition}

\subsubsection{Proposed Technique \& Features}

It presents a new texture analysis method for personal identification, which is based on iris patterns. It is made up of image acquisition, image preprocessing, feature extraction, and decision stages and uses MATLAB. It is realized in the Windows platform using the Delphi compiler.

\subsubsection{Advantages}

The proposed system mainly focuses on the preprocessing of iris images and in the feature extraction stages and includes the major properties of an iris recognition system, such as scale and rotation invariance.

\subsubsection{Limitations}

This method faces issues regarding eyelash elimination and lack of training stage.

\subsection{Recognition of Human Iris Patterns for Biometric Identification}

\subsubsection{Proposed Technique \& Features}

In this paper, the author considers iris recognition as the most difficult and fundamental step in an iris recognition system. The techniques are more reliably described by the iris when the iris borders are approximated by two circles, resulting in better recognition performance. The normalization, encoding, and matching methods are implemented in this paper.

\subsubsection{Advantages}

It improves and increases detection accuracy and efficiency.

Since it deals with raw derivative information, it does not suffer from thresholding problems.

\subsubsection{Limitations}

The processing time is not evaluated and is a very complex method.

It can fail where there is noise in the eye image, such as from reflections.

\subsection{Feature Extraction Techniques for Recognition of Iris Images \\ 2.4.1 Proposed Technique \& Features}

In this paper, the author considers iris recognition as the most difficult and fundamental step in an iris recognition system. When two circles approximate the iris borders, the approaches are more correctly defined by the iris, resulting in better recognition performance. The normalization, encoding, and matching methods are implemented in this paper.

\subsubsection{Advantages}

It uses the phase-based method and Feature Extraction method for obtaining higher accuracy in recognition also the iris feature vector length is much smaller than the other methods.

All the strategies suggested in this paper save time in the processing and extraction of features in comparison with the known existence methods.

\subsubsection{Limitations}


Volume 8 Issue 12 December 2020

In this system, intensity variation and processing time are not evaluated.

The difficulty of the extraction of features influences the program complexity and the processing speed of systems for iris recognition.

\subsection{Wireless attendance management system based on iris recognition 2.5.1 Proposed Technique \& Features}

In this paper, a wireless iris recognition system technique solves the issues regarding spurious attendance and the difficulties of laying a corresponding network. It helps to provide the user's attendances more easily and effectively. The process is implemented using Daugman's algorithm.

\subsubsection{Advantages}

The performance of this system meets the requirements of the daily attendance management in institutions and enterprises.

It is designed based on the automatic iris recognition module and RF wireless module.

\subsubsection{Limitations:}

It is difficult to set the transmission lines where the topography is bad.

\subsection{Retina Based Biometric} Authentication System

\subsubsection{Proposed Technique \& Features}

This paper proposes the process for iris recognition including pre-processing, feature extraction, and features matching. In this process, either blood vessel features or non-blood vessel features are used. The proposed system implements a fuzzy circular Hough transform. For extraction of blood vessels, it uses MLSEC (multi-local level set extrinsic curvature).

\subsubsection{Advantages}

This paper improves recognition rates, equal error rates, processing time, and the number of images taken in different methods.

\subsubsection{Limitations}

If a person is suffering from some eye diseases like hard glaucoma, cataracts and so on then the identification process will be difficult.

\subsection{Iris Recognition Systems Technical Overview}

In this paper, segmentation isolates the iris region from the whole eye. For the correct processing of the recognition system, this stage is very essential. Daugman proposed an Integro-differential operator for locating the inner and outer boundaries of iris, additionally as the upper and lower eyelids.

\subsubsection{Advantages}

In recent years, iris identification has been actively researched.

This paper presents an up-to-date analysis of major studies into iris recognition.

\subsubsection{Limitations}

Iris segmentation may make the process difficult.

\section{CONCLUSION AND FUTURE SCOPE}

We studied publications on iris-recognition systems that related to our project. In these proposed research works, iris recognition has not been implemented for a real-time application that aims at solving a real-world issue. We have synthesized the study from these papers to learn about existing methodologies and to initiate work in our proposed system. In future, we aim at developing an automated system for attendance monitoring based on iris recognition systems. The systems will reduce manual work and provide other functionalities such as information retrieval, alerts and notifications along with updating the attendance database.

\section{ACKNOWLEDGMENTS}

We are grateful to the faculty members at JAIN (Deemed-to-be) University for their extensive support in our literary research for this paper. Likewise, our sincere gratitude are for our ever supportive parents who are always there for us through thick and thin.

\section{REFERENCES}

[1]. Javier, Paulín \& Alberto, Lara \& María, Romero \& Jiménez-Hernández, Hugo. (2019). "Implementation of the Hough Transform for Iris Detection and Segmentation." Advances in Molecular Imaging. 6-18. 10.4236/ami.2019.91002.

[2]. Gürkan, Güray \& Akan, Aydin. (2006). "Texture Analysis Based Iris Recognition.” 6.

[3]. E. Ali, E. S. Ahmed, and A. F. AM. li, "Recognition of Human Iris Patterns for Biometric Identification,” J. Eng. Appl. Sci., vol. 54, no. 6, pp. 635-651, 2019. 
Volume 8 Issue 12 December 2020

[4]. Alice Nithya. A. And Lakshmi, C, "Feature Extraction Techniques for Recognition of iris images." 2020

[5]. Mazumdar, Jarina. (2018). "Retina Based Biometric Authentication System: A Review." International Journal of Advanced Research in Computer $\quad$ Science. $\quad 9$. 711-718. 10.26483/ijarcs.v9i1.5322.

[6]. Kadry, Seifedine \& Smaili, Mohamad. (2010). "Wireless Attendance Management System Based on Iris Recognition." Scientific Research and Essays. 5. 1428-1435.

[7]. Omidiora, Elijah \& Adegoke, B \& A.s, Falohun \& Ojo, John. (2015). "Iris Recognition Systems: Technical Overview.” 3. 63-72.

[8]. Olatinwo, S.O. \& Shoewu, Oluwagbemiga \& Omitola, Olusegun. (2013). "Iris Recognition Technology: Implementation, Application, and Security Consideration." The Pacific Journal of Science and Technology. 14. 228-233.
[9]. Kalaiselvi, S. \& Anandha Jothi, Rajendran \& Palanisamy, Vellaiyan. (2018). "Biometric security with iris recognition techniques: A review." International Journal of Pure and Applied Mathematics. 118. 567-571.

[10]. Bhawna, Chouhan \& Shukla, Shailja. (2011). "Iris Recognition System using canny edge detection for Biometric Identification." International Journal of Engineering Science and Technology. 3. 